\title{
PREVALENCE AND PATTERN OF SUBSTANCE ABUSE AMONG DRIVERS IN PUNJAB AND HIMACHAL PRADESH
}

\author{
Dhruvendra Lal', Tanvir Kaur Sidhu², P. P. S. Coonar ${ }^{3}$ \\ ${ }_{13}^{\text {rd }}$ Year Postgraduate Student, Department of Community Medicine, AIMSR, Bathinda, Punjab. \\ 2Professor, Department of Community Medicine, AIMSR, Bathinda, Punjab. \\ 3 Professor and HOD, Department of Community Medicine, AIMSR, Bathinda, Punjab.
}

ABSTRACT

\section{BACKGROUND}

Substance abuse as an epidemic among people in India has assumed alarming dimensions. Substance abuse refers to the harmful or hazardous use of psychoactive substances including alcohol and illicit drugs. Driving Under the Influence (DUI) of drugs is an unspoken danger on the roads. The effects of specific drugs differ depending on how they act in the brain. There is one death every minute in India due to road accidents. Drunken driving is one of the leading causes of road fatalities.

Aim- To study the pattern and prevalence and pattern of substance abuse among drivers in Punjab and Himachal Pradesh.

\section{MATERIALS AND METHODS}

A community-based, cross-sectional study was conducted among native drivers of vehicles including goods carriers. One district of each state, i.e. Punjab and Himachal Pradesh were chosen out of convenience.

\section{RESULTS}

A total of 328 drivers were included in the study. Alcohol was the most commonly used substance abuse in both states. Prevalence of alcohol in Punjab and Himachal was $40 \%$ and $64 \%$, respectively. Age and education was significantly associated with the use of illicit substance of abuse.

\section{CONCLUSION}

This study showed that quite a number of drivers do substance abuse and there were significant associations between various risk factors, social factors and substance abuse among the drivers.

\section{KEYWORDS}

Substance Abuse, Punjab, Himachal Pradesh, Drivers, Prevalence, Pattern, Risk Factors.

HOW TO CITE THIS ARTICLE: Lal D, Sidhu TK, Coonar PPS. Prevalence and pattern of substance abuse among drivers in Punjab and Himachal Pradesh. J. Evolution Med. Dent. Sci. 2017;6(48):3686-3693, DOI: 10.14260/Jemds/2017/796

\begin{abstract}
BACKGROUND
Substance abuse as an epidemic among people in India has assumed alarming dimensions. Changing cultural values, increasing economic stress and dwindling supportive bonds are leading to initiation into substance use. ${ }^{1}$ June $26^{\text {th }}$ is celebrated as International Day against Drug Abuse and Illicit Trafficking every year.

Substance abuse refers to the harmful or hazardous use of psychoactive substances including alcohol and illicit drugs. Psychoactive substance use can lead to dependence syndrome- a cluster of behavioural, cognitive and physiological phenomena that develop after repeated substance use and that typically include a strong desire to take the drug, difficulties in controlling its use, persisting in its use despite harmful consequences, a higher priority given to drug use than to other activities and obligations, increased tolerance and sometimes a physical withdrawal state. ${ }^{2}$
\end{abstract}

Financial or Other, Competing Interest: None.

Submission 05-05-2017, Peer Review 03-06-2017

Acceptance 08-06-2017, Published 15-06-2017.

Corresponding Author:

Dr. Dhruvendra Lal,

$3^{\text {rd }}$ Year Postgraduate Student,

Department of Community Medicine,

AIMSR, Bathinda, Barnala Road,

Bhucho Khurd-151001, Punjab.

E-mail: laldhruv_1987@yahoo.com,drdhruvlal@gmail.com

DOI: $10.14260 /$ jemds $/ 2017 / 796$
According to a 2011 report on drug abuse and alcoholism in Punjab by the Ministry of Youth Affairs and Sports, "a 40 percent of Punjabi youth in the age group of 15 to 25 years have fallen prey to drugs." 3 Golden Crescent- they call it. It is the route through which the drug cartels enter India, mainly Punjab. The clandestine land routes of Iran, Afghanistan and Pakistan collectively constitute the Golden Crescent, also called the silk route of drugs. Heroin, poppy husk, opium and synthetic drugs are the four kinds of drugs entering India. ${ }^{4}$ Household surveys have shown that 65 percent households in Majha and Doaba have at least one drug user, similarly 64 percent families in Malwa have at least one drug abuse case. 5 In 2013, the BSF has recovered nearly $390 \mathrm{kgs}$ of Heroin, almost four times the amount seized in $2008 .{ }^{6}$ In 2013 - 14, the Customs Department of Punjab has seized $74.672 \mathrm{kgs}$ valued at Rs. 374.05 crores found concealed in the Samjhauta Express and Goods Train coming from Pakistan. ${ }^{7}$ As per a survey conducted by the Guru Nanak Dev University, some 73.5 percent of the state's youth between 16 - 35 years were drug addicts. ${ }^{8}$

On the other hand, Malana and Kasol (District Kullu) have been preferred destinations for many, who visit the place in huge numbers. Cannabis has always been grown in this area, but was meant for personal consumption and has great level of social acceptance. The local culture, which is guided to a great extent by belief in 'devta' (Almost every village in Himachal has their own local deities and all major decisions are taken with their permission), treats cannabis as 'shiv ji ki 
buti' and does not see cannabis production as something wrong. ${ }^{9}$ Despite police efforts, illegal drug trade is rising in Himachal Pradesh; 1,914 cases were registered in the state since 2011 (up to April 30, 2014) under Narcotics Drugs and Psychotropic Substances Act (NDPS). Narcotics Control Bureau, North Zone, Chandigarh, estimated around 60 tonnes of charas is produced in the state annually. The report said that cannabis plantations exist in Parbati valley, Avgal Thach area, some $15 \mathrm{kms}$ from Malana, Brona Nullah area towards Khirganga, Tosh and Barshaini. ${ }^{10}$

Driving Under the Influence (DUI) of drugs is an unspoken danger on the roads. There has been an alarming increase in the number of drivers causing road traffic accidents with many of them being under the influence of one or the other substances of abuse. The effects of specific drugs differ depending on how they act in the brain. For example, marijuana can slow reaction time, impair judgment of time and distance, and decrease coordination. Drivers who have used cocaine or methamphetamine can be aggressive and reckless when driving. Certain kinds of sedatives called benzodiazepines can cause dizziness and drowsiness. All of these impairments can lead to vehicle crashes. ${ }^{11}$ It is important that car accidents caused by drugged driving due to not only illegal substances, but prescribed and nonprescribed over-the-counter medications - are stopped and that the dangers of this kind of DUI are taken seriously. ${ }^{12}$ According to a report published by NDTV over 1,37,000 people were killed in road accidents in 2013 alone, that is more than the number of people killed in all our wars put together with 16 children dying on Indian roads daily. There is one death every minute in India due to road accidents. Drunken driving is one of the leading causes of road fatalities.13,14 According to another report published in The Hindu, a vast 70 percent of fatalities in road accidents were due to drunken driving, thus questions are being raised on the mushrooming growth of liquor vends along the highways.

This study therefore is aimed at determining the prevalence and pattern of substance use among drivers in Dist. Bathinda, Punjab and Dist. Kullu, Himachal Pradesh and the factors influencing the same.

\section{Aim and Objective}

Aim

To study the prevalence and pattern of substance abuse among drivers in Punjab and Himachal Pradesh.

\section{Objectives}

1. To study the prevalence and pattern of substance abuse among drivers.

2. Compare the prevalence and pattern of substance abuse between Dist. Bathinda, Punjab and Dist. Kullu, Himachal Pradesh.

3. To study association of various risk factors with substance abuse.

4. To assess knowledge, attitude and risk behaviours associated with substance abuse and driving.

\section{MATERIALS AND METHODS}

\section{Study Design}

A community-based, cross-sectional study was conducted among native drivers of all vehicles including goods carriers, at halting places. One district of each state, i.e. Punjab and Himachal Pradesh were chosen out of convenience. The participants were included in the study in both the districts till the desired sample size was obtained.

\section{Unit of Study}

Drivers of various vehicles. Clearance from AIMSR Research Committee and Adesh University Ethics Committee was taken before the conduction of study. Ethical clearance from Civil Surgeon (Chief Medical Officer), Zonal Hospital, Dist. Kullu, Himachal Pradesh was also taken and predesigned questionnaire was introduced to the participants.

\section{Sample Size}

According to a study conducted by Makanjuola $A B$, Department of Behavioural Sciences, University of Ilorin Teaching Hospital, Ilorin, Nigeria use prevalence of psychoactive substances was alcohol (71.6\%), tobacco (69.8\%), caffeine (50.9\%), amphetamine (2.4\%) and sedatives $(6.9 \%) .15$

\section{To Calculate Sample Size following Formula was used-} $\mathrm{N}=\left[\mathrm{Z}^{2} \mathrm{P}(1-\mathrm{P})\right] /\left[\mathrm{d}^{2}\right]$

Talking prevalence of alcohol usage, $71.6 \%$ according to the above study, our sample size came out to be a minimum of 325.25

Verbal consent was taken from all the drivers and anonymity was maintained.

\section{Participants}

- Inclusion Criteria: Subjects who were willing to participate in the study.

- Exclusion Criteria: Subjects who were not willing to participate in the study.

\section{Sampling Method}

Convenient sampling was used in this study, in which two districts were chosen according to the working place. One day a week was randomly selected to conduct the study at various halting places in both the states. The study was conducted for two months in each in District Bathinda and District Kullu and all the subjects who gave consent to participate in the study on that particular day were included. The study was conducted from 9 am to $5 \mathrm{pm}$ and was continued till the desired sample size was obtained. Thus, the place of study was chosen as per convenience and the days were chosen by randomly and all the subjects willing to participate were included in the study on these days.

\section{Statistical Methods}

Percentages, frequencies, mean, median, mode, standard deviation, Chi Square test, Univariate logistic regression and Multivariate logistic regression were used to analyse the results on this study. IBM SPSS Version 20 was used to analyse the study.

\section{RESULTS}

A total of 328 drivers were included in the study. This includes 164 participants from District Bathinda, Punjab and 164 from District Kullu, Himachal Pradesh. The demographic data of the participants of this study is given in Table 1; 
$70.7 \%$ of the participants from Punjab were in the age range of 18 to 28 years as compared to $44.5 \%$ of Himachal Pradesh; 54.9\% from Punjab and 61.6\% from Himachal Pradesh belonged to rural areas and the remaining from urban sector. The illiteracy was comparatively less in Himachal with $6.7 \%$ participants being illiterate. There were only $22.6 \%$ graduates or above in Himachal; $81.1 \%$ of the participants from Punjab and $69.5 \%$ from Himachal had 4 or more family members.

Table 2 shows the professional details of all the drivers from both the states. There were a few participants who were not holding any driving license, both in Punjab and Himachal (4.9\% and $2.4 \%$, respectively). There were more number of challans of the vehicles in Himachal as compared to Punjab (28.7\% and $11.6 \%$, respectively). On the contrary, more number of driving license were revoked in Punjab (5.5\%) as compared to $1.8 \%$ (3 participants) in Himachal.

Alcohol was the most commonly used substance abuse in both states. Prevalence of alcohol in Punjab and Himachal was $40 \%$ and $64 \%$, respectively. Tobacco smoking was more prevalent in Himachal with prevalence of $43.3 \%$ as compared to $9.1 \%$ in Punjab. Tobacco chewing was also more common in Himachal than Punjab, prevalence being $14 \%$ and $6.7 \%$ respectively. Marijuana users were more in the Himachal with 19 of the participants as the users and with a prevalence of $11.6 \%$. Bhukki/Afeem/Opium was more prevalent in Himachal. Prevalence of opium in Himachal and Punjab being $10.4 \%$ and $6.7 \%$, respectively. Heroin users were equitably distributed in both the states with 4 users in each. Figure 1 shows the prevalence of various substance abuses.

Table 3 shows associations of various variable with substance abuse. The study found out that the drivers in the age group of 28 - 48 years were significantly ( $p$ value $<0.001$ ) more into substance abuse as compared to other age groups. The drivers with income $<10,000$ Rs. were more into substance abuse, while drivers who were educated only up to high school were more likely associated with substance abuse as compared to those who were more educated. Higher number of family members and drivers of heavy vehicles were also significantly associated with substance abuse; 66 drivers got themselves challan'd in the past one year, out of which 53 were some kind of substance abusers, i.e. about $80 \%$ of the drivers who got challan'd quoted yes to substance abuse.

Table 4 shows the pattern and behaviour of drivers in two states and many variables were found to have significant differences in their behaviour and pattern of substance abuse. Table 5 shows regression analysis of various variables with substance abuse as dependent variable. As the age increased, the proneness into substance abuse increased. Drivers aged 48 to 68 years were 48.22 times more prone to substance abuse as compared to 18 to 28 years drivers when analysed using multivariate logistic regression, ( $\mathrm{p}$ value $<0.001$ ) and 10.855 times more susceptible when using univariate logistic regression ( $\mathrm{p}$ value < 0.001). Drivers with primary and middle school education were also 93.595 ( $p$ value $<0.001$ ) and 15.550 ( $\mathrm{p}$ value $=0.010$ ) times more prone to substance abuse as compared to illiterate drivers using multivariate logistic regression. Bus and truck drivers were 15.39 times more into use of these substances of abuse as compared to three wheel/auto-rickshaw drivers, who were taken as reference $(p$ value $=0.013$ ). Drivers with no driving license were 100.407 times more into abuse as compared to twowheeled license holders ( $p$ value $=0.003$ ). Driver who were challan'd in the past one year were 16.294 times more into abuse ( $p$ value $<0.001$ ) using multivariate regression analysis and 3.608 times more using univariate logistic regression ( $p$ value $<0.001$ ). Univariate logistic regression of multiple sex partners as independent variable showed that the ones indulging into the same were 4.078 times more into substance abuse.

\begin{tabular}{|c|c|c|c|c|}
\hline $\begin{array}{l}\text { Age in } \\
\text { Years }\end{array}$ & $\begin{array}{c}\text { Punjab } \\
(n=164)\end{array}$ & $\begin{array}{c}\% \\
\text { (Punjab) }\end{array}$ & $\begin{array}{c}\text { H.P. } \\
(n=164)\end{array}$ & $\begin{array}{c}\% \\
\text { (HP) }\end{array}$ \\
\hline $18-28$ & 116 & 70.7 & 73 & 44.5 \\
\hline $28-48$ & 40 & 24.4 & 61 & 37.2 \\
\hline $48-68$ & 6 & 3.7 & 27 & 16.5 \\
\hline$>68$ & 2 & 1.2 & 3 & 1.8 \\
\hline \multicolumn{5}{|l|}{ Sex } \\
\hline Male & 143 & 87.2 & 154 & 93.9 \\
\hline Female & 21 & 12.8 & 10 & 6.1 \\
\hline \multicolumn{5}{|l|}{ Area } \\
\hline Rural & 90 & 54.9 & 101 & 61.6 \\
\hline Urban & 74 & 45.1 & 63 & 38.4 \\
\hline \multicolumn{5}{|l|}{ Religion } \\
\hline Sikh & 92 & 56.1 & 6 & 3.7 \\
\hline Hindu & 62 & 37.8 & 131 & 79.9 \\
\hline Muslim & 4 & 2.4 & 14 & 8.5 \\
\hline Christian & 2 & 1.2 & 7 & 4.3 \\
\hline Buddhist & 0 & 0 & 3 & 1.8 \\
\hline Others & 4 & 2.4 & 3 & 1.8 \\
\hline \multicolumn{5}{|l|}{$\begin{array}{c}\text { Income Per } \\
\text { Month in } \\
\text { Rupees }\end{array}$} \\
\hline$>30,000$ & 57 & 34.8 & 32 & 19.5 \\
\hline $\begin{array}{c}20,000 \text { to } \\
29,999\end{array}$ & 56 & 34.1 & 13 & 7.9 \\
\hline $\begin{array}{c}10,000 \text { to } \\
19,999\end{array}$ & 35 & 21.3 & 26 & 15.9 \\
\hline 0 to 9999 & 16 & 9.8 & 93 & 56.7 \\
\hline \multicolumn{5}{|l|}{ Education } \\
\hline Illiterate & 14 & 8.5 & 11 & 6.7 \\
\hline Can ready only & 7 & 4.3 & 4 & 2.4 \\
\hline $\begin{array}{c}\text { Can read and } \\
\text { write }\end{array}$ & 13 & 7.9 & 9 & 5.5 \\
\hline $\begin{array}{c}\text { Primary } \\
\text { School }\end{array}$ & 12 & 7.3 & 26 & 15.9 \\
\hline Middle School & 25 & 15.2 & 11 & 6.7 \\
\hline High School & 29 & 17.7 & 66 & 40.2 \\
\hline $\begin{array}{c}\text { Graduate and } \\
\text { above }\end{array}$ & 64 & 39 & 37 & 22.6 \\
\hline \multicolumn{5}{|l|}{$\begin{array}{c}\text { Family } \\
\text { Members }\end{array}$} \\
\hline Stay alone & 2 & 1.2 & 7 & 4.3 \\
\hline 2 Members & 7 & 4.3 & 3 & 1.8 \\
\hline 3 Members & 22 & 13.4 & 40 & 24.4 \\
\hline 4 or More & 133 & 81.1 & 114 & 69.5 \\
\hline
\end{tabular}




\begin{tabular}{|c|c|c|c|c|}
\hline $\begin{array}{c}\text { Number of } \\
\text { Children }\end{array}$ & & & & \\
\hline 1 Child & 32 & 19.5 & 44 & 26.8 \\
\hline 2 Children & 70 & 42.7 & 79 & 48.2 \\
\hline 3 Children & 19 & 11.6 & 15 & 9.1 \\
\hline 4 or More & 6 & 3.7 & 20 & 12.2 \\
\hline None & 37 & 22.6 & 6 & 3.7 \\
\hline $\begin{array}{c}\text { Keep away } \\
\text { from Family }\end{array}$ & & & & \\
\hline $\begin{array}{c}\text { Meet Family } \\
\text { Daily }\end{array}$ & 64 & 39 & 37 & 22.6 \\
\hline For Days & 66 & 40.2 & 86 & 52.4 \\
\hline For Weeks & 8 & 4.9 & 28 & 17.1 \\
\hline For Months & 26 & 15.9 & 13 & 7.9 \\
\hline \multicolumn{7}{|c|}{ Table 1. Demographic Profile of Participants } \\
\hline
\end{tabular}

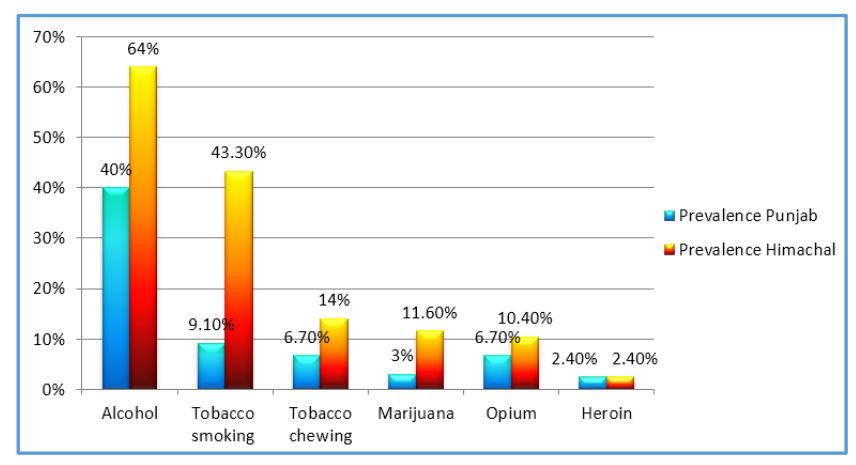

Figure 1. Prevalence of Various Substance Abuse in Himachal and Punjab

\begin{tabular}{|c|c|c|c|c|}
\hline & $\begin{array}{c}\text { Punjab } \\
(n=164)\end{array}$ & \begin{tabular}{|c|}
$\%$ \\
(Punjab)
\end{tabular} & $\begin{array}{c}\text { HP } \\
(n=164)\end{array}$ & $\begin{array}{c}\% \\
\text { (HP) }\end{array}$ \\
\hline \multicolumn{5}{|l|}{$\begin{array}{c}\text { Type of Driving } \\
\text { License }\end{array}$} \\
\hline Two-Wheeler & 5 & 3 & 4 & 2.4 \\
\hline LMV & 116 & 70.7 & 77 & 47 \\
\hline HMV & 35 & 21.3 & 79 & 48.2 \\
\hline No License & 8 & 4.9 & 4 & 2.4 \\
\hline \multicolumn{5}{|l|}{ Type of Vehicle } \\
\hline Three-Wheeler & 5 & 3 & 8 & 4.9 \\
\hline Private Car & 108 & 65.9 & 57 & 34.8 \\
\hline Taxi & 11 & 6.7 & 40 & 24.4 \\
\hline Bus & 8 & 4.9 & 7 & 4.3 \\
\hline Truck & 30 & 18.3 & 11 & 6.7 \\
\hline $\begin{array}{l}\text { Heavy Vehicle } \\
\text { (>6 Wheels) }\end{array}$ & 2 & 1.2 & 41 & 25 \\
\hline \multicolumn{5}{|l|}{$\begin{array}{l}\text { Duration of } \\
\text { Driving }\end{array}$} \\
\hline$<1$ Year & 3 & 1.8 & 6 & 3.7 \\
\hline 1 to 5 Years & 83 & 50.6 & 25 & 15.2 \\
\hline 5 to 10 Years & 54 & 32.9 & 27 & 16.5 \\
\hline$>10$ Years & 24 & 14.6 & 106 & 64.6 \\
\hline \multicolumn{5}{|l|}{ Challan History } \\
\hline Yes & 19 & 11.6 & 47 & 28.7 \\
\hline No & 145 & 88.4 & 117 & 71.3 \\
\hline \multicolumn{5}{|l|}{ License Revoked } \\
\hline Yes & 9 & 5.5 & 3 & 1.8 \\
\hline No & 155 & 94.5 & 161 & 98.2 \\
\hline \multicolumn{5}{|l|}{\begin{tabular}{|c|} 
Expenditure per \\
Month \\
on Substance \\
Abuse \\
\end{tabular}} \\
\hline $\begin{array}{c}\text { Do not do } \\
\text { substance abuse }\end{array}$ & 84 & 51.2 & 52 & 31.7 \\
\hline 2000 to 3999 & 45 & 27.5 & 80 & 48.7 \\
\hline 2000 to 3999 & 19 & 11.6 & 17 & 10.4 \\
\hline 4000 to 5999 & 14 & 8.5 & 7 & 4.3 \\
\hline More than 6000 & 2 & 1.2 & 8 & 4.9 \\
\hline \multicolumn{5}{|c|}{ Table 2. Professional Profile of Drivers } \\
\hline
\end{tabular}

\begin{tabular}{|c|c|c|c|c|c|}
\hline \multirow{3}{*}{\multicolumn{2}{|c|}{ Variables }} & \multicolumn{4}{|c|}{ Substance Abuse } \\
\hline & & \multirow{3}{*}{$\begin{array}{c}\text { Yes } \\
\% \\
83 \\
(25.3 \%)\end{array}$} & \multirow{3}{*}{\begin{tabular}{|c|} 
No \\
\% \\
106 \\
$(32.3 \%)$ \\
\end{tabular}} & \multirow{3}{*}{\begin{tabular}{|c|} 
Total \\
$\%$ \\
189 \\
$(57.6 \%)$ \\
\end{tabular}} & \multirow{2}{*}{ P Value } \\
\hline & & & & & \\
\hline \multirow{5}{*}{ Age } & $\begin{array}{c}18 \text { to } 28 \\
\text { Years }\end{array}$ & & & & \multirow{5}{*}{$\mathrm{p}<0.001$} \\
\hline & $\begin{array}{c}28 \text { to } 48 \\
\text { Years }\end{array}$ & $\begin{array}{c}75 \\
(22.9 \%) \\
\end{array}$ & $\begin{array}{c}26 \\
(7.9 \%) \\
\end{array}$ & $\begin{array}{c}101 \\
(30.8 \%)\end{array}$ & \\
\hline & $\begin{array}{c}48 \text { to } 68 \\
\text { Years }\end{array}$ & $\begin{array}{c}29 \\
(8.8 \%)\end{array}$ & $\begin{array}{c}4 \\
(1.2 \%)\end{array}$ & $\begin{array}{c}33 \\
(10.1 \%)\end{array}$ & \\
\hline & $\begin{array}{c}>68 \\
\text { Years }\end{array}$ & $\begin{array}{c}5 \\
(1.5 \%)\end{array}$ & $\begin{array}{c}0 \\
(0 \%)\end{array}$ & $\begin{array}{c}5 \\
(1.5 \%) \\
\end{array}$ & \\
\hline & Total & $\begin{array}{c}192 \\
(58.5 \%)\end{array}$ & $\begin{array}{c}136 \\
(41.5 \%)\end{array}$ & $\begin{array}{c}328 \\
(100.0 \%)\end{array}$ & \\
\hline \multirow{3}{*}{ Area } & Rural & $\begin{array}{c}109 \\
(33.2 \%)\end{array}$ & $\begin{array}{c}82 \\
(25.0 \%) \\
\end{array}$ & $\begin{array}{c}191 \\
(58.2 \%)\end{array}$ & \multirow{3}{*}{$\begin{array}{l}p>0.05 \\
(0.524)\end{array}$} \\
\hline & Urban & $\begin{array}{c}83 \\
(25.3 \%)\end{array}$ & $\begin{array}{c}54 \\
(16.5 \%)\end{array}$ & $\begin{array}{c}137 \\
(41.8 \%)\end{array}$ & \\
\hline & Total & $\begin{array}{c}192 \\
(58.5 \%)\end{array}$ & $\begin{array}{c}136 \\
(41.5 \%)\end{array}$ & $\begin{array}{c}328 \\
(100.0 \%)\end{array}$ & \\
\hline \multirow{5}{*}{$\begin{array}{c}\text { Income } \\
\text { per } \\
\text { month } \\
\text { in } \\
\text { Rupees }\end{array}$} & $>30,000$ & $\begin{array}{c}49 \\
(14.9 \%)\end{array}$ & $\begin{array}{c}40 \\
(12.2 \%)\end{array}$ & $\begin{array}{c}89 \\
(27.1 \%)\end{array}$ & \multirow{5}{*}{$\begin{array}{l}p<0.05 \\
(0.026)\end{array}$} \\
\hline & $\begin{array}{c}20,000 \\
\text { to } \\
29,999 \\
\end{array}$ & $\begin{array}{c}40 \\
(12.2 \%)\end{array}$ & $\begin{array}{c}29 \\
(8.8 \%) \\
\end{array}$ & $\begin{array}{c}69 \\
(21.0 \%)\end{array}$ & \\
\hline & $\begin{array}{c}10,000 \\
\text { to } \\
19,999\end{array}$ & $\begin{array}{c}28 \\
(8.5 \%)\end{array}$ & $\begin{array}{c}33 \\
(10.1 \%)\end{array}$ & $\begin{array}{c}61 \\
(18.6 \%)\end{array}$ & \\
\hline & $\begin{array}{c}0 \text { to } \\
9999\end{array}$ & $\begin{array}{c}75 \\
(22.9 \%)\end{array}$ & $\begin{array}{c}34 \\
(10.4 \%)\end{array}$ & $\begin{array}{c}109 \\
(33.2 \%)\end{array}$ & \\
\hline & Total & $\begin{array}{c}192 \\
(58.5 \%)\end{array}$ & $\begin{array}{c}136 \\
(41.5 \%)\end{array}$ & $\begin{array}{c}328 \\
(100.0 \%)\end{array}$ & \\
\hline \multirow{8}{*}{$\begin{array}{c}\text { Educa- } \\
\text { tion }\end{array}$} & Illiterate & $\begin{array}{c}15 \\
(4.6 \%) \\
\end{array}$ & $\begin{array}{c}10 \\
(3.0 \%)\end{array}$ & $\begin{array}{c}25 \\
(7.6 \%) \\
\end{array}$ & \multirow{8}{*}{$p<0.001$} \\
\hline & $\begin{array}{c}\text { Can Read } \\
\text { Only }\end{array}$ & $7(2.1 \%)$ & $4(1.2 \%)$ & $\begin{array}{c}11 \\
(3.4 \%) \\
\end{array}$ & \\
\hline & $\begin{array}{c}\text { Can Read } \\
\text { and } \\
\text { Write } \\
\end{array}$ & $10(.3 .0 \%)$ & $\begin{array}{c}12 \\
(3.7 \%)\end{array}$ & $\begin{array}{c}22 \\
(6.7 \%)\end{array}$ & \\
\hline & $\begin{array}{c}\text { Primary } \\
\text { School }\end{array}$ & $\begin{array}{c}35 \\
(10.7 \%)\end{array}$ & $3(0.9 \%)$ & $\begin{array}{c}38 \\
(11.6 \%)\end{array}$ & \\
\hline & $\begin{array}{l}\text { Middle } \\
\text { School } \\
\end{array}$ & $24(7.3 \%)$ & $\begin{array}{c}12 \\
(3.7 \%) \\
\end{array}$ & $\begin{array}{c}36 \\
(11.0 \%) \\
\end{array}$ & \\
\hline & $\begin{array}{c}\text { High } \\
\text { School }\end{array}$ & $\begin{array}{c}55 \\
(16.8 \%)\end{array}$ & $\begin{array}{c}40 \\
(12.2 \%)\end{array}$ & $\begin{array}{c}95 \\
(29.0 \%)\end{array}$ & \\
\hline & $\begin{array}{c}\text { Graduate } \\
\text { and } \\
\text { Above }\end{array}$ & $\begin{array}{c}46 \\
(14.0 \%)\end{array}$ & $\begin{array}{c}55 \\
(16.8 \%)\end{array}$ & $\begin{array}{c}101 \\
(30.8 \%)\end{array}$ & \\
\hline & Total & $\begin{array}{c}192 \\
(58.5 \%)\end{array}$ & $\begin{array}{c}136 \\
(41.5 \%) \\
\end{array}$ & $\begin{array}{c}328 \\
(100.0 \%)\end{array}$ & \\
\hline \multirow{3}{*}{$\begin{array}{c}\text { Number } \\
\text { of } \\
\text { Family } \\
\text { Members }\end{array}$} & $\begin{array}{l}\text { Stay } \\
\text { Alone }\end{array}$ & $\begin{array}{c}4 \\
(1.2 \%)\end{array}$ & $\begin{array}{c}5 \\
(1.5 \%)\end{array}$ & $\begin{array}{c}9 \\
(2.7 \%)\end{array}$ & \multirow[b]{3}{*}{$\begin{array}{l}\mathrm{p}<0.01 \\
(0.002)\end{array}$} \\
\hline & 2 & $\begin{array}{c}8 \\
(2.4 \%) \\
\end{array}$ & $\begin{array}{c}2 \\
(0.6 \%) \\
\end{array}$ & $\begin{array}{c}10 \\
(3.0 \%) \\
\end{array}$ & \\
\hline & 3 & $\begin{array}{c}48 \\
(14.6 \%) \\
\end{array}$ & $\begin{array}{c}14 \\
(4.3 \%) \\
\end{array}$ & $\begin{array}{c}62 \\
(18.9 \%) \\
\end{array}$ & \\
\hline
\end{tabular}




\begin{tabular}{|c|c|c|c|c|c|}
\hline & $\begin{array}{l}4 \text { or } \\
\text { More }\end{array}$ & $\begin{array}{c}132 \\
(40.2 \%) \\
\end{array}$ & $\begin{array}{c}115 \\
(35.1 \%) \\
\end{array}$ & $\begin{array}{c}247 \\
(75.3 \%) \\
\end{array}$ & \\
\hline & Total & $\begin{array}{c}192 \\
(58.5 \%)\end{array}$ & $\begin{array}{c}136 \\
(41.5 \%)\end{array}$ & $\begin{array}{c}328 \\
(100.0 \%)\end{array}$ & \\
\hline \multirow{7}{*}{$\begin{array}{l}\text { Type of } \\
\text { Vehicle } \\
\text { Driven }\end{array}$} & $\begin{array}{c}\text { Three- } \\
\text { Wheeler }\end{array}$ & $\begin{array}{c}7 \\
(2.1 \%)\end{array}$ & $\begin{array}{c}6 \\
(1.8 \%)\end{array}$ & $\begin{array}{c}13 \\
(4.0 \%)\end{array}$ & \multirow{7}{*}{$<0.001$} \\
\hline & $\begin{array}{c}\text { Private } \\
\text { Car }\end{array}$ & $\begin{array}{c}89 \\
(27.1 \%)\end{array}$ & $\begin{array}{c}76 \\
(23.2 \%)\end{array}$ & $\begin{array}{c}165 \\
(50.3 \%)\end{array}$ & \\
\hline & Taxi & $\begin{array}{c}26 \\
(7.9 \%) \\
\end{array}$ & $\begin{array}{c}25 \\
(7.6 \%) \\
\end{array}$ & $\begin{array}{c}51 \\
(15.5 \%) \\
\end{array}$ & \\
\hline & Bus & $\begin{array}{c}15 \\
(4.6 \%) \\
\end{array}$ & $\begin{array}{c}0 \\
(0.0 \%)\end{array}$ & $\begin{array}{c}15 \\
(4.6 \%) \\
\end{array}$ & \\
\hline & Truck & $19(5.8 \%)$ & $\begin{array}{c}22 \\
(6.7 \%)\end{array}$ & $\begin{array}{c}41 \\
(12.5 \%) \\
\end{array}$ & \\
\hline & $\begin{array}{c}\text { Heavy } \\
\text { Vehicle } \\
\quad>6 \\
\text { Wheels }) \\
\end{array}$ & $\begin{array}{c}36 \\
(11.0 \%)\end{array}$ & $\begin{array}{c}7 \\
(2.1 \%)\end{array}$ & $\begin{array}{c}43 \\
(13.1 \%)\end{array}$ & \\
\hline & Total & $\begin{array}{c}192 \\
(58.5 \%)\end{array}$ & $\begin{array}{c}136 \\
(41.5 \%)\end{array}$ & $\begin{array}{c}328 \\
(100.0 \%)\end{array}$ & \\
\hline \multirow{3}{*}{$\begin{array}{l}\text { Challan } \\
\text { History }\end{array}$} & Yes & $\begin{array}{c}53 \\
(16.2 \%)\end{array}$ & $\begin{array}{c}13 \\
(4.0 \%)\end{array}$ & $\begin{array}{c}66 \\
(20.1 \%)\end{array}$ & \multirow[b]{3}{*}{$\mathrm{p}<0.001$} \\
\hline & No & $\begin{array}{c}139 \\
(42.4 \%)\end{array}$ & $\begin{array}{c}123 \\
(37.5 \%)\end{array}$ & $\begin{array}{c}262 \\
(79.9 \%)\end{array}$ & \\
\hline & Total & $\begin{array}{c}192 \\
(58.5 \%) \\
\end{array}$ & $\begin{array}{c}136 \\
(41.5 \%) \\
\end{array}$ & $\begin{array}{c}328 \\
(100.0 \%)\end{array}$ & \\
\hline \multirow{5}{*}{$\begin{array}{c}\text { Duration } \\
\text { One } \\
\text { Keeps } \\
\text { Away } \\
\text { from } \\
\text { Family }\end{array}$} & $\begin{array}{l}\text { Meet } \\
\text { Family } \\
\text { Daily } \\
\end{array}$ & $\begin{array}{c}55 \\
(16.8 \%)\end{array}$ & $\begin{array}{c}46 \\
(14.0 \%)\end{array}$ & $\begin{array}{c}101 \\
(30.8 \%)\end{array}$ & \multirow{5}{*}{$\begin{array}{l}p>0.05 \\
(0.059)\end{array}$} \\
\hline & For Days & $\begin{array}{c}89 \\
(27.1 \%) \\
\end{array}$ & $\begin{array}{c}63 \\
(19.2 \%) \\
\end{array}$ & $\begin{array}{c}152 \\
(46.3 \%)\end{array}$ & \\
\hline & $\begin{array}{c}\text { For } \\
\text { Weeks }\end{array}$ & $\begin{array}{c}28 \\
(8.5 \%) \\
\end{array}$ & $\begin{array}{c}8 \\
(2.4 \%)\end{array}$ & $\begin{array}{c}36 \\
(11.0 \%)\end{array}$ & \\
\hline & $\begin{array}{c}\text { For } \\
\text { Months }\end{array}$ & $20(6.1 \%)$ & $\begin{array}{c}19 \\
(5.8 \%)\end{array}$ & $\begin{array}{c}39 \\
(11.9 \%)\end{array}$ & \\
\hline & & $\begin{array}{c}192 \\
(58.5 \%)\end{array}$ & $\begin{array}{c}136 \\
(41.5 \%)\end{array}$ & $\begin{array}{c}328 \\
(100.0 \%)\end{array}$ & \\
\hline
\end{tabular}

\begin{tabular}{|c|c|c|c|c|c|}
\hline Variable & & Punjab & HP & Total & P value \\
\hline \multirow{3}{*}{$\begin{array}{c}\text { Had } \\
\text { Alcohol in } \\
\text { the Past } 1 \\
\text { Year }\end{array}$} & Yes & $\begin{array}{c}80 \\
(24.4 \%)\end{array}$ & $\begin{array}{c}112 \\
(34.1 \%)\end{array}$ & $\begin{array}{c}192 \\
(58.5 \%)\end{array}$ & \multirow{3}{*}{$\mathrm{P}<0.001$} \\
\hline & No & $\begin{array}{c}84 \\
(25.6 \%) \\
\end{array}$ & $\begin{array}{c}52 \\
(15.9 \%) \\
\end{array}$ & $\begin{array}{c}136 \\
(41.5 \%)\end{array}$ & \\
\hline & Total & $\begin{array}{c}164 \\
(50 \%)\end{array}$ & $\begin{array}{c}164 \\
(50 \%) \\
\end{array}$ & $\begin{array}{c}328 \\
(100 \%) \\
\end{array}$ & \\
\hline \multirow{6}{*}{$\begin{array}{l}\text { Frequency } \\
\text { of Alcohol }\end{array}$} & Daily & $\begin{array}{c}7 \\
(2.1 \%)\end{array}$ & $\begin{array}{c}30 \\
(9.1 \%)\end{array}$ & $\begin{array}{c}37 \\
(11.3 \%)\end{array}$ & \multirow{6}{*}{$\mathrm{P}<0.001$} \\
\hline & $\begin{array}{c}2-4 \\
\text { Times a } \\
\text { Week }\end{array}$ & $\begin{array}{c}43 \\
(13.1 \%)\end{array}$ & $\begin{array}{c}57 \\
(17.4 \%)\end{array}$ & $\begin{array}{c}100 \\
(30.5 \%)\end{array}$ & \\
\hline & $\begin{array}{c}\text { Once } \\
\text { Weekly }\end{array}$ & $9(2.7 \%)$ & $16(4.9 \%)$ & $\begin{array}{c}25 \\
(7.6 \%)\end{array}$ & \\
\hline & $\begin{array}{c}\text { Monthly } \\
\text { or Less }\end{array}$ & $\begin{array}{c}21 \\
(6.4 \%)\end{array}$ & $\begin{array}{c}9 \\
(2.7 \%)\end{array}$ & $\begin{array}{c}30 \\
(9.1 \%)\end{array}$ & \\
\hline & Never & \begin{tabular}{|c|}
84 \\
$(25.6 \%)$ \\
\end{tabular} & $\begin{array}{c}52 \\
(15.9 \%) \\
\end{array}$ & $\begin{array}{c}136 \\
(41.5 \%) \\
\end{array}$ & \\
\hline & Total & $\begin{array}{c}164 \\
(50 \%)\end{array}$ & $\begin{array}{c}164 \\
(50 \%)\end{array}$ & $\begin{array}{c}328 \\
(100 \%)\end{array}$ & \\
\hline \multirow{3}{*}{\begin{tabular}{|c|} 
Use \\
Pharmaceu \\
tical Drug \\
Abuse
\end{tabular}} & Yes & $3(0.9 \%)$ & $4(1.2 \%)$ & $7(2.1 \%)$ & \multirow{3}{*}{$\begin{array}{c}p>0.05 \\
(=0.702)\end{array}$} \\
\hline & No & $\begin{array}{c}161 \\
(49.1 \%) \\
\end{array}$ & $\begin{array}{c}160 \\
(48.8 \%) \\
\end{array}$ & $\begin{array}{c}321 \\
(97.9 \%) \\
\end{array}$ & \\
\hline & Total & $\begin{array}{c}164 \\
(50 \%)\end{array}$ & $\begin{array}{c}164 \\
(50 \%)\end{array}$ & $\begin{array}{c}328 \\
(100 \%)\end{array}$ & \\
\hline
\end{tabular}

\begin{tabular}{|c|c|c|c|c|c|}
\hline \multirow{3}{*}{$\begin{array}{l}\text { Used I/V } \\
\text { Drugs }\end{array}$} & Yes & $\begin{array}{c}157 \\
(47.9 \%)\end{array}$ & $\begin{array}{c}24 \\
(7.3 \%) \\
\end{array}$ & \begin{tabular}{|c|}
181 \\
$(55.2 \%)$ \\
\end{tabular} & \multirow{3}{*}{$\mathrm{p}<0.001$} \\
\hline & No & $\begin{array}{c}7 \\
(2.1 \%)\end{array}$ & $\begin{array}{c}140 \\
(42.7 \%)\end{array}$ & $\begin{array}{c}147 \\
(44.8 \%)\end{array}$ & \\
\hline & Total & $\begin{array}{c}164 \\
(50 \%)\end{array}$ & $\begin{array}{c}164 \\
(50 \%)\end{array}$ & $\begin{array}{c}328 \\
(100 \%) \\
\end{array}$ & \\
\hline \multirow{3}{*}{$\begin{array}{l}\text { Sighted for } \\
\text { Impaired } \\
\text { Driving }\end{array}$} & Yes & $3(0.9 \%)$ & $3(0.9 \%)$ & $6(1.8 \%)$ & \multirow[b]{3}{*}{$\mathrm{p}>0.05$} \\
\hline & No & $\begin{array}{c}161 \\
(49.1 \%)\end{array}$ & $\begin{array}{c}161 \\
(49.1 \%)\end{array}$ & \begin{tabular}{|c|}
322 \\
$(98.2 \%)$
\end{tabular} & \\
\hline & Total & $\begin{array}{c}164 \\
(50 \%)\end{array}$ & $\begin{array}{c}164 \\
(50 \%)\end{array}$ & $\begin{array}{c}328 \\
(100 \%)\end{array}$ & \\
\hline \multirow{3}{*}{$\begin{array}{c}\text { You/Some } \\
\text { one } \\
\text { Injured } \\
\text { because of } \\
\text { Substance } \\
\text { Abuse in } \\
\text { last } 1 \text { Year } \\
\end{array}$} & Yes & $\begin{array}{c}15 \\
(4.6 \%)\end{array}$ & $11(3.4 \%)$ & $\begin{array}{c}26 \\
(7.9 \%)\end{array}$ & \multirow[b]{3}{*}{$\begin{array}{c}p>0.05 \\
(=0.414)\end{array}$} \\
\hline & No & $\begin{array}{c}149 \\
(45.4 \%) \\
\end{array}$ & $\begin{array}{c}153 \\
(46.6 \%) \\
\end{array}$ & $\begin{array}{c}302 \\
(92.1 \%) \\
\end{array}$ & \\
\hline & Total & $\begin{array}{c}164 \\
(50 \%)\end{array}$ & $\begin{array}{c}164 \\
(50 \%)\end{array}$ & $\begin{array}{c}328 \\
(100 \%)\end{array}$ & \\
\hline \multirow{3}{*}{\begin{tabular}{|c|} 
Road \\
Accident \\
under \\
Substance \\
Abuse in \\
last 1 Year
\end{tabular}} & Yes & $\begin{array}{c}8 \\
(2.4 \%) \\
\end{array}$ & $\begin{array}{c}22 \\
(6.7 \%)\end{array}$ & $\begin{array}{c}30 \\
(9.1 \%)\end{array}$ & \multirow{3}{*}{$\begin{array}{c}\mathrm{p}<0.01 \\
(=0.007)\end{array}$} \\
\hline & No & $\begin{array}{c}156 \\
(47.6 \%)\end{array}$ & $\begin{array}{c}142 \\
(43.3 \%)\end{array}$ & $\begin{array}{c}298 \\
(90.9 \%) \\
\end{array}$ & \\
\hline & Total & $\begin{array}{c}164 \\
(50 \%) \\
\end{array}$ & $\begin{array}{c}164 \\
(50 \%) \\
\end{array}$ & \begin{tabular}{|c|}
328 \\
$(100 \%)$ \\
\end{tabular} & \\
\hline \multirow{3}{*}{$\begin{array}{l}\text { Committed } \\
\text { any Crime } \\
\text { under } \\
\text { Substance } \\
\text { Abuse in } \\
\text { last } 1 \text { Year }\end{array}$} & Yes & $5(1.5 \%)$ & $31(9.5 \%)$ & $\begin{array}{c}36 \\
(11 \%)\end{array}$ & \multirow[b]{3}{*}{$\mathrm{p}<0.001$} \\
\hline & No & $\begin{array}{c}159 \\
(48.5 \%) \\
\end{array}$ & $\begin{array}{c}133 \\
(40.5 \%) \\
\end{array}$ & $\begin{array}{c}292 \\
(89 \%) \\
\end{array}$ & \\
\hline & Total & $\begin{array}{c}164 \\
(50 \%) \\
\end{array}$ & $\begin{array}{c}164 \\
(50 \%) \\
\end{array}$ & \begin{tabular}{|c|}
328 \\
$(100 \%)$ \\
\end{tabular} & \\
\hline \multirow{3}{*}{$\begin{array}{l}\text { Multiple } \\
\text { Sex } \\
\text { Partners }\end{array}$} & Yes & $\begin{array}{c}6 \\
(1.8 \%)\end{array}$ & $\begin{array}{c}41 \\
(12.5 \%)\end{array}$ & $\begin{array}{c}47 \\
(14.3 \%)\end{array}$ & \multirow{3}{*}{$\mathrm{p}<0.001$} \\
\hline & No & $\begin{array}{c}158 \\
(48.2) \\
\end{array}$ & $\begin{array}{c}123 \\
(37.5 \%) \\
\end{array}$ & \begin{tabular}{|c|}
281 \\
$(85.7 \%)$ \\
\end{tabular} & \\
\hline & Total & $\begin{array}{c}164 \\
(50 \%)\end{array}$ & $\begin{array}{c}164 \\
(50 \%) \\
\end{array}$ & $\begin{array}{c}328 \\
(100 \%) \\
\end{array}$ & \\
\hline \multirow{3}{*}{ Gambling } & Yes & $\begin{array}{c}35 \\
(10.7 \%) \\
\end{array}$ & $18(5.5 \%)$ & $\begin{array}{c}53 \\
(16.2 \%) \\
\end{array}$ & \multirow{3}{*}{$\begin{array}{c}p<0.05 \\
(=0.011)\end{array}$} \\
\hline & No & $\begin{array}{c}129 \\
(39.3 \%) \\
\end{array}$ & $\begin{array}{c}146 \\
(44.5 \%) \\
\end{array}$ & \begin{tabular}{|c|}
275 \\
$(83.5 \%)$ \\
\end{tabular} & \\
\hline & Total & $\begin{array}{c}164 \\
(50 \%) \\
\end{array}$ & $\begin{array}{c}164 \\
(50 \%) \\
\end{array}$ & \begin{tabular}{|c|}
328 \\
$(100 \%)$ \\
\end{tabular} & \\
\hline \multirow{3}{*}{$\begin{array}{c}\text { Had H/O } \\
\text { TB }\end{array}$} & Yes & $\begin{array}{c}4 \\
(1.2 \%) \\
\end{array}$ & $\begin{array}{c}7 \\
(2.1 \%) \\
\end{array}$ & $\begin{array}{c}11 \\
(3.4 \%)\end{array}$ & \multirow{3}{*}{$\begin{array}{l}p>0.05 \\
(0.358)\end{array}$} \\
\hline & No & $\begin{array}{c}160 \\
(48.8 \%)\end{array}$ & $\begin{array}{c}157 \\
(47.9 \%)\end{array}$ & \begin{tabular}{|c|}
317 \\
$(96.6 \%)$
\end{tabular} & \\
\hline & Total & $\begin{array}{c}164 \\
(50 \%)\end{array}$ & $\begin{array}{c}164 \\
(50 \%)\end{array}$ & \begin{tabular}{|c|}
328 \\
$(100 \%)$
\end{tabular} & \\
\hline$T$ & 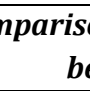 & $\begin{array}{l}\text { of Pat } \\
\text { een th }\end{array}$ & $\begin{array}{l}\text { rn and Be } \\
\text { Two Stat }\end{array}$ & haviour & Drivers \\
\hline
\end{tabular}




\begin{tabular}{|c|c|c|c|c|c|c|}
\hline \multirow[b]{2}{*}{ Covariates } & \multicolumn{2}{|c|}{ Substance Abuse } & \multicolumn{2}{|c|}{ Univariate Logistic Regression } & \multicolumn{2}{|c|}{ Multivariate Logistic Regression } \\
\hline & Yes & No & P value & $\begin{array}{c}\text { OR } \\
\text { (95\% Confidence } \\
\text { Interval) }\end{array}$ & $P$ value & $\begin{array}{c}\text { OR } \\
\text { (95\% Confidence } \\
\text { Interval) }\end{array}$ \\
\hline Age & & & $<0.001$ & & $<0.001$ & \\
\hline 18 to 28 years & $\begin{array}{c}83 \\
(25.3 \%)\end{array}$ & $\begin{array}{c}106 \\
(32.3 \%)\end{array}$ & & 1 & & 1 \\
\hline 28 to 48 years & $75(22.9 \%)$ & $26(7.9 \%)$ & $<0.001$ & $3.684(2.167-6.262)$ & 0.175 & $1.879(0.756-4.672)$ \\
\hline 48 to 68 years & $34(10.4 \%)$ & $4(1.2 \%)$ & $<0.001$ & $10.855(3.705-31.810)$ & $<0.001$ & $48.222(7.053-329.717)$ \\
\hline \multicolumn{7}{|l|}{ Area } \\
\hline Urban & $83(25.3 \%)$ & $54(16.5 \%)$ & & 1 & & 1 \\
\hline Rural & $109(33.2 \%)$ & $82(25.0 \%)$ & 0.524 & $1.156(0.740-1.807)$ & 0.021 & $3.247(1.198-8.804)$ \\
\hline Income & & & 0.029 & & 0.141 & \\
\hline$>30,000$ & $49(14.9 \%)$ & $40(12.2 \%)$ & & 1 & & 1 \\
\hline $20,000-29,999$ & $40(12.2 \%)$ & $29(8.8 \%)$ & 0.714 & $1.126(0.597-2.124)$ & 0.331 & $1.783(0.556-5.713)$ \\
\hline $10,000-19,999$ & $28(8.5 \%)$ & $33(10.1 \%)$ & 0.271 & $0.693(0.360-1.332)$ & 0.113 & $0.373(0.110-1.265)$ \\
\hline$<10,000$ & $75(22.9 \%)$ & $34(10.4 \%)$ & 0.048 & $1.801(1.006-3.222)$ & 0.912 & $1.070(0.322-3.557)$ \\
\hline Education & & & 0.002 & & $<0.001$ & \\
\hline Illiterate & $15(4.6 \%)$ & $10(3.0 \%)$ & & 1 & & 1 \\
\hline Can Read Only & $7(2.1 \%)$ & $4(1.2 \%)$ & 0.837 & $1.167(0.269-5.054)$ & 0.016 & 21.857 (1.772-269.589) \\
\hline Can Read and Write & $10(3.0 \%)$ & $12(3.7 \%)$ & 0.320 & $0.556(0.174-1.771)$ & 0.412 & $0.397(0.044-3.614)$ \\
\hline Primary School & $35(10.7 \%)$ & $3(0.9 \%)$ & 0.005 & $7.778(1.871-32.337)$ & $<0.001$ & $93.595(7.819-1120.346)$ \\
\hline Middle School & $24(7.3 \%)$ & $12(3.7 \%)$ & 0.594 & $1.333(0.463-3.843)$ & 0.010 & 15.550 (1.909-126.637) \\
\hline High School & $55(16.8 \%)$ & $40(12.2 \%)$ & 0.849 & $0.917(0.373-2.250)$ & 0.195 & $3.360(0.537-21.030)$ \\
\hline $\begin{array}{l}\text { Graduate and } \\
\text { Above }\end{array}$ & $46(14.0 \%)$ & $55(16.8 \%)$ & 0.199 & $0.558(0.229-1.359)$ & 0.472 & $2.113(0.276-16.200)$ \\
\hline $\begin{array}{c}\text { Number of Family } \\
\text { Members }\end{array}$ & & & 0.004 & & 0.211 & \\
\hline Stay Alone & $4(1.2 \%)$ & $5(1.5 \%)$ & & 1 & & 1 \\
\hline 2 Members & $8(2.4 \%)$ & $2(0.6 \%)$ & 0.121 & $5.000(0.655-38.152)$ & 0.852 & $1.364(0.052-35.857)$ \\
\hline 3 Members & $48(14.6 \%)$ & $14(4.3 \%)$ & 0.048 & $4.286(1.012-18.148)$ & 0.961 & $1.067(0.078-14.527)$ \\
\hline 4 or More & $132(40.2 \%)$ & $115(35.1 \%)$ & 0.597 & $1.435(0.376-5.470)$ & 0.400 & $0.357(0.032-3.932)$ \\
\hline Vehicle Type & & & 0.14 & & $<0.001$ & \\
\hline 3-Wheeler & $7(2.1 \%)$ & $6(1.8 \%)$ & & 1 & & 1 \\
\hline Car/Taxi & $115(35.1 \%)$ & $101(30.8 \%)$ & 0.966 & $0.976(0.318-2.999)$ & 0.834 & $1.241(0.165-9.349)$ \\
\hline Bus/Truck & $70(21.3 \%)$ & $29(8.8 \%)$ & 0.225 & $2.069(0.640-6.687)$ & 0.013 & 15.139 (1.769-129.535) \\
\hline License Type & & & 0.132 & & $<0.001$ & \\
\hline Two-Wheeler & $7(2.1 \%)$ & $2(0.6 \%)$ & & 1 & & 1 \\
\hline LMV & $103(31.4 \%)$ & $90(27.4 \%)$ & 0.170 & $0.327(0.066-1.614)$ & 0.341 & $3.348(0.279-40.243)$ \\
\hline HMV & $74(22.6 \%)$ & $40(12.2 \%)$ & 0.440 & $0.529(0.105-2.665)$ & 0.512 & $0.438(0.037-5.151)$ \\
\hline No License & $8(2.4 \%)$ & $4(1.2 \%)$ & 0.579 & $0.571(0.079-4.128)$ & 0.003 & 100.407 (4.663-2161.942) \\
\hline \multicolumn{7}{|l|}{$\begin{array}{l}\text { Challan'd in the } \\
\text { last } 1 \text { Year }\end{array}$} \\
\hline No & $139(42.4 \%)$ & $123(37.5 \%)$ & & 1 & & 1 \\
\hline Yes & $53(16.2 \%)$ & $13(4.0 \%)$ & $<0.001$ & $3.608(1.877-6.934)$ & $<0.001$ & $16.294(4.704-56.442)$ \\
\hline \begin{tabular}{|c|}
$\begin{array}{c}\text { How Long Drivers } \\
\text { are Away from } \\
\text { Home }\end{array}$ \\
\end{tabular} & & & 0.084 & & 0.180 & \\
\hline \begin{tabular}{|l|} 
Meet Family Daily \\
\end{tabular} & $55(16.8 \%)$ & $46(14.0 \%)$ & & 1 & & 1 \\
\hline For Days & $89(27.1 \%)$ & $63(19.2 \%)$ & 0.519 & $1.182(0.711-1.963)$ & 0.411 & $1.571(0.535-4.613)$ \\
\hline For Weeks & $28(8.5 \%)$ & $8(2.4 \%)$ & 0.16 & $2.927(1.217-7.043)$ & 0.704 & $1.353(0.284-6.438)$ \\
\hline For Months & $20(6.1 \%)$ & $19(5.8 \%)$ & 0.880 & $(0.420-1.845)$ & 0.136 & $0.333(0.078-1.415)$ \\
\hline \multicolumn{7}{|l|}{$\begin{array}{c}\text { Multiple Sex } \\
\text { Partners }\end{array}$} \\
\hline No & $153(46.6 \%)$ & $128(39.0 \%)$ & & 1 & & 1 \\
\hline Yes & $39(11.9 \%)$ & $8(2.4 \%)$ & 0.001 & $4.078(1.840-9.042)$ & 0.542 & $1.589(0.359-7.038)$ \\
\hline \multicolumn{7}{|l|}{\begin{tabular}{|c|}
$\begin{array}{c}\text { Friends or Family } \\
\text { do Substance } \\
\text { Abuse }\end{array}$ \\
\end{tabular}} \\
\hline No & $84(25.6 \%)$ & $117(35.7 \%)$ & & 1 & & 1 \\
\hline Yes & $108(32.9 \%)$ & $19(5.8 \%)$ & $<0.001$ & $7.917(4.512-13.894)$ & $<0.001$ & 32.213 (11.489-90.317) \\
\hline & Table 5. K & ression And & (Com & d for Punjab and $\mathrm{Hi}$ & 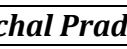 & \\
\hline
\end{tabular}




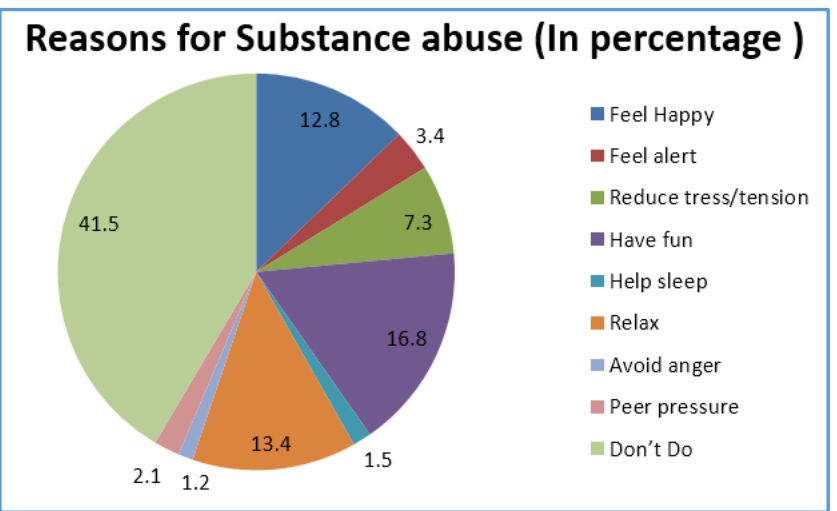

Figure 2. Reasons for Substance Abuse (In Percentage)

\section{DISCUSSION}

Historically, roadside surveys have been conducted as a means to obtain an objective, scientifically valid estimate of the extent of driving after drinking within specified geographic and temporal parameters. Drink or use drugs and drive, and the results can be deadly. In addition to thousands of injuries, several hundred people die every year because of the same. This study gave a snapshot of pattern and prevalence of substance abuse among drivers in Punjab and Himachal, and it also provided inputs on the factors associated with substance abuse.

This study found out that alcohol was the most common substance abuse used both in Punjab (40\%) as well as Himachal Pradesh (60\%). Tobacco smoking was more prevalent in Himachal (43.3\%). Tobacco chewing, Marijuana and Opium was also more prevalent in Himachal Pradesh as seen in Figure II. Age and income of drivers was significantly associated with substance abuse. Drivers who attended school were also into substance abuse. There was a significant association between the drivers who got challan'd and those who used substance abuse. A similar study was conducted by Nigeriaky Makanjuola et al, which showed that most psychoactive substances among Long Distance Vehicle (LDV) drivers were mild stimulants (31.9\%), cigarette (30.4\%), anabolic steroid (27.5\%) and alcohol (15.9\%).16

Another study conducted by L Morrison et al on personal and situational influences on drink driving showed that drivers with lower socioeconomic status or with no school qualification or those who were dependent on alcohol or marijuana at age 21 were significantly more likely to report drunken driving incidence at age 26.17

In our study, the prevalence of substance abuse increased as the age group advances. Drivers from both rural and urban sectors took part in the study; however, the substance abuse was higher in the rural background. It was observed that most of the drivers had family size of 4 or more members with many of them having 2 children. Most of the drivers from both states kept away from their families for days. The participating drivers were mostly holding Light Motor Vehicle (LMV) driving license. Ashish Das et al in his review of studies concluded that as many as 23 studies reported use of alcohol by drivers during driving. The study analysed that $2 \%-33 \%$ of injured and $6 \%-48 \%$ of killed Road Traffic Crash victims had consumed alcohol or were under the influence of drugs. ${ }^{18}$ Another study Nilambar Jha et al stated that $14.9 \%$ of Road Traffic Accident (RTA) victims coming to hospitals had consumed alcohol. ${ }^{19}$
The problem of substance abuse remains a grave issue in both the states. The study found out that there was more prevalence of substance abuse in Himachal Pradesh than in Punjab. Our study also found out significant association between various associated risk behaviours and substance abuse among drivers. Drivers with no license, heavy vehicle drivers (Bus/truck) and those drivers who had family history of substance abuse were significantly more prone to substance abuse. More such studies are needed to be conducted to get clearer picture of this menace, not only amongst drivers but also among others in the community.

\section{CONCLUSION}

This study showed that quite a number of drivers do substance abuse and there were significant associations between various risk factors, social factors and substance abuse among the drivers. Blood alcohol levels should be regularly monitored by the traffic police and strict enforcement of Motor Vehicle Act on drunk driving should be done. Those found under influence of any substance abuse should be given strict and swift punishment. Driver education courses should be made more approachable and taught seriously, as they are not only responsible for their own lives but also of their co-passengers and the pedestrians. For safety of the drivers under influence of any substance abuse must be regularly identified, removed from the road, provide effective treatment and bring in them the behaviour change before permitting them to drive. The need of the hour is- "Drive Slow- Reach Safely" and not the race with devil to reach the tomb prematurely.

\section{REFERENCES}

[1] Nadeem A, Rubeena B, Agarwal VK, et al. Substance abuse in India. Pravara Med Rev 2009;1(4):4-6.

[2] WHO. Substance abuse 2016.

[3] Ministry of youth affairs and sports, awareness and education for prevention of drug abuse \& alcoholism. In: Punjab, New Delhi: Nehru Yuva Kendra Sangathan. Social justice report 2011. Available from:

http://www.nyks.org/PDF\%20File/SocialJusticReport .pdf.

[4] Golden crescent - the route through which drugs are making their way into Punjab. Available from: http://www.indiatimes.com/news/india/goldencrescent-the-route-through-which-drugs-are-makingtheir-way-into-punjab-256556.html.

[5] Jones RK, Shinar D, Walsh JM. State of knowledge of drug-impaired driving, DOT Report \#809 642, national highway traffic safety administration, September, 2003:p120. Available from: https://www.google.co.in/search?sclient=psyab\&site $=$ \&source $=h p \& q=2 . \% 09 J$ ones + RK\%2C + Shinar $+\mathrm{D} \% 2 \mathrm{C}+$ Walsh + JM. + State + of + Knowledge + of + DrugImpaired+Driving\%2C+DOT+Report+\%23809+642\% $2 \mathrm{C}+$ National+Highway+Traffic+Safety+Administration $\% 2 \mathrm{C}+$ September $\% 2 \mathrm{C}+2003+\% 28120+$ pgs $\% 29 .+\&$ btn $\mathrm{K}=$ Google+Search\#q=http:\%2F\%2Fclient.webdweb.co m\%2Frgics\%2Fsites\%2Fdefault\%2Ffiles\%2FDrug\%2 520Addiction\%2520in\%2520Punjab.pdf+. 
[6] Drug trafficking reaches new high in Punjab. Latest news \& updates at daily news \& analysis 2016. Available from:

http://www.dnaindia.com/india/report-drugtrafficking-reaches-new-high-in-punjab-1903083.

[7] Customs Commissionerate (Prev). Amritsar 2016. Available from: http://amritsarcustoms.gov.in/english/pressrelase.ht m.

[8] Targeted interventions for truckers operational guidelines NACO 2016. Available from: http://naco.gov.in/upload/Policies\%20\&\%20Guidelin es/28,

\%20Targeted $\% 20$ Interventions $\% 20$ for\%20Truckers \%20-\%200perational\%20Guidelines.pdf.

[9] Hash'tag Himachal: how this beautiful mountain state turned into a narcotics den. Firstpost 2016. Available from: http://www.firstpost.com/india/himachalpradesh-narcotics-malana-village-marijuana-opium2747436.html.

[10] Drug trade rising in Himachal. Times of India. The Times of India 2016. Available from: http://timesofindia.indiatimes.com/city/chandigarh/ Drug-trade-rising-inHimachal/articleshow/37490147.cms.

[11] Drug facts: drugged driving. National Institute on Drug Abuse 2016. Available from: https://www.drugabuse.gov/publications/drugfacts/ drugged-driving.

[12] Drugged driving is a top cause of auto accidents. Law Offices of Michael Pines, APC 2016. Available from: https://seriousaccidents.com/legal-advice/topcauses-of-car-accidents/driving-under-the-influencedui-of-drugs.
[13] Road accident statistics in India. Road Safety 2014. Available from: http://sites.ndtv.com/roadsafety/important-featureto-you-in-your-car.

[14] Mohan D, Tiwari G, Bhalla K. Road safety in India status report. TRIPP Transport Research and Injury Prevention Programme 2015. Available from: http://tripp.iitd.ernet.in/road_safety_in_India_status_r eport.pdf.

[15] Makanjuola AB, Aina OF, Onigbogi L. Alcohol and other psychoactive substance use among tanker drivers in Lagos, Nigeria. European Scientific Journal 2014;10(5):545-59.

[16] Makanjuola BA, Oyeleke S, Akande T. Psychoactive substance use among long distance vehicle drivers in Ilorin, Nigeria. Nigerian Journal of Psychiatry 2007;5(1).

[17] Morrison L, Begg D, Langley J. Personal and situational influences on drink driving and sober driving among a cohort of young adults. Injury Prevention 2002;8(2):111-5.

[18] Das A, Gjerde H, Gopalan SS, et al. Alcohol, drugs, and road traffic crashes in India: a systematic review. Traffic Injury Prevention 2012;13(6):544-53.

[19] Jha N, Srinivasa DK, Roy G, et al. Epidemiological study of road traffic accident cases: a study from South India. Indian Journal of Community Medicine 2004;29(1): 20-4. 